\title{
Energy accumulation in mechanical resonance and its use in drive systems of impact machines
}

\author{
Wieslaw Fiebig ${ }^{1} \cdot$ Jakub Wróbel $^{1}$
}

Received: 4 February 2020 / Accepted: 19 February 2020 / Published online: 2 March 2020

(c) The Author(s) 2020

\begin{abstract}
An innovative method utilizing the phenomenon of mechanical resonance in machine drive systems of impact machines has been described. Energy accumulated in mechanical resonance is typically multiple times larger than the continuously supplied excitation energy. The amount of accumulated energy depends on the amplitude of vibration, mass and stiffness of system and present energy dissipation mechanisms. This form of energy can be extracted periodically each time after the amplitude of vibration reaches its maximum value. This effect could be implemented in drive systems of periodically operating machines, such as punching machines and presses. Significant reduction of energy demand in machines that use mechanical resonance in comparison with conventional machines was confirmed by means of simulations and experiments. Accumulation of the energy at mechanical resonance and its sequential extraction were presented together with a new prototype resonance punching press.
\end{abstract}

Keywords Mechanical resonance $\cdot$ Drive systems $\cdot$ Impact machines $\cdot$ Energy savings

\section{Introduction}

Resonance in mechanical systems is almost always considered as an undesirable phenomenon. Stronger vibrations cause significantly higher dynamic loads on mechanical systems components as well as accelerated fatigue. Critical or cycling loads can impact the structural integrity of mechanical systems, e.g., civil structures, rotational machinery, etc. On the other hand, resonance was successfully applied in other fields of engineering and science such as medical diagnostics, telecommunications, laser technology and acoustics [1-3].

An example of using mechanical resonance to increase efficiency of a machine is the resonance hammer drilling (RHD) [4] where the phenomenon of resonance is implemented to increase the efficiency of drilling in hard materials. RHD utilizes the vibration generated during the cutting process to exert a harmonic load on the drill bit as well as on

Wieslaw Fiebig

wieslaw.fiebig@pwr.edu.pl

1 Faculty of Mechanical Engineering, Wroclaw University of Science and Technology, Lukasiewicza 7/9, 50-37 Wrocław, Poland a hammer attached to the bit. At resonance, the bit is subject to cyclic impacts generated by the hammer.

Mechanical resonance is commonly applied in drive systems of vibratory conveyors [5]. Experimental investigations show that electromagnetic excitation of vibratory conveyors resonant modes is advantageous due to the reduced amount of energy needed to maintain desirable conveyor oscillations.

Industrial robots [6] should not utilize large actuators to accelerate and deaccelerate operational elements but provide conditions for the kinetic-potential energy form transformation. In robots of that type, energy economies of up to $56 \%$ have been achieved due to the use of resonance energy. Positive effects of mechanical resonance implementation are also visible in drive systems of small flying objects in form of micro-propulsion systems [7].

Mechanical resonance is also used microscale scavenging devices, where vibrations are used to power sensors, onboard devices and MEMSs [8, 9]. Transformation of vibration energy can take place due to electromagnetic or mechanical events, e.g., via a generator. Energy harvesting was described in a number of publications [8-11]. Vibrational energy is harvestable in microscale from vehicle suspension systems, railway tracks, motion of humans, etc. [11-13]. It was observed by several researchers that the harvesting 
efficiency is increased at resonance conditions of the harvester generating elements [14-16]. Researchers also used the resonance vibration amplitude magnification to improve the energy generation properties of harvesters $[17,18]$.

\section{Theoretical background}

Figure 1 shows an oscillator comprising a mass $M$ connected to two spring elements. The excitation force is generated by a rotationally coupled rod mass $m$. The mass $M$ is mounted on a slide between two springs. Each spring is characterized by the stiffness coefficient $k / 2$ and the damping coefficient $c / 2$.

The kinetic energy of the oscillator KE is the sum of the kinetic energy of the cart (mass $M$ ) and the kinetic energy of the rotating rod:

$$
\begin{aligned}
\mathrm{KE}= & \frac{1}{2} M \dot{x}^{2}+\frac{1}{2} m\left[\dot{x}^{2}-2 l \dot{x} \dot{\varphi} \sin (\varphi)+l \dot{\varphi}^{2} \sin ^{2}(\varphi)\right] \\
& +\frac{1}{2} m \dot{\varphi}^{2} l^{2} \cos ^{2}(\varphi)+I_{c} \dot{\varphi}^{2}
\end{aligned}
$$

where $I_{c}=\frac{1}{3} m(2 l)^{2}$-momentum of inertia of the rotating rod.

The potential energy of the system PE is defined by the energy accumulated in the system in form of spring deflection and the angular position of the rotating rod:

$\mathrm{PE}=\frac{1}{2} k x^{2}+m g l \sin (\varphi)$.

The equations of motion have been derived with the Lagrange method from following equations:

$\frac{\mathrm{d}}{\mathrm{d} t}\left(\frac{\partial L}{\partial \dot{q}_{i}}\right)-\frac{\partial L}{\partial q_{i}}+\frac{\partial D}{\partial \dot{q}_{i}}=Q_{i}$

where $\boldsymbol{L}=\mathrm{KE}-\mathrm{PE}$-Lagrangian function, $q_{i}$ corresponds to the generalized coordinates and $\mathrm{D}$ to the energy dissipation function, $Q_{i}$-generalized force.

$(M+m) \ddot{x}-m l\left(\ddot{\varphi} \sin \varphi+\dot{\varphi}^{2} \cos \varphi\right)+c \dot{x}+k x=0$

$\left(I_{c}+m l^{2}\right) \ddot{\varphi}-m l \ddot{x} \sin \varphi+m g l \cos \varphi=M_{m}$ where $M_{m}$-torque on the electric motor.

Assuming that the rotating rod has a constant angular velocity $\omega$, no angular acceleration is present and therefore the equation of motion, obtained with the Lagrange method, takes the following final form:

$\ddot{x}(M+m)+k x+c \dot{x}=\omega^{2} m l \cos (\varphi)$.

The total energy $\mathrm{TE}_{m}$ of the rotating rod mass is the sum of the kinetic energy of the rod mass $m$, the rotational kinetic energy and the variable potential energy of the rod mass. The damping ratio of the system is assumed to be reasonably small and was not included in the energy description for the purpose of theoretical considerations; therefore,

$\mathrm{TE}_{m}=\frac{1}{2} m v_{c x}^{2}+\frac{1}{2} I_{c} \dot{\varphi}^{2}+m g l \sin (\varphi)$

where

$v_{c x}^{2}=(\dot{x}-l \dot{\varphi} \sin (\varphi))^{2}+\dot{\varphi}^{2} l^{2} \cos ^{2}(\varphi)$.

The total energy $\mathrm{TE}_{M}$ of the cart mass $M$ can be expressed as

$T E_{M}=\frac{1}{2} M \dot{x}^{2}+\frac{1}{2} k x^{2}$.

The parameters of the model used in simulations are shown in Table 1.

The damping factor has been determined experimentally on a physical model presented in Fig. 2. Figure 2a shows the displacement amplitude of the mass $\mathrm{M}$ at resonance. It can be seen that the amplitude reaches its maximal value after about $13 \mathrm{~s}$.

Table 1 Data for simulation

\section{Data for simulation}

Oscillating mass $M[\mathrm{~kg}]$

4.555

Rotating rod mass $m[\mathrm{~kg}]$

0.593

Spring stiffness $k[\mathrm{~N} / \mathrm{m}]$

750

Damping coefficient $c$ [Ns/m]

3

Radius of gravity point of the rotating mass $l[\mathrm{~m}]$

0.108

Fig. 1 A single-mass mechanical oscillator

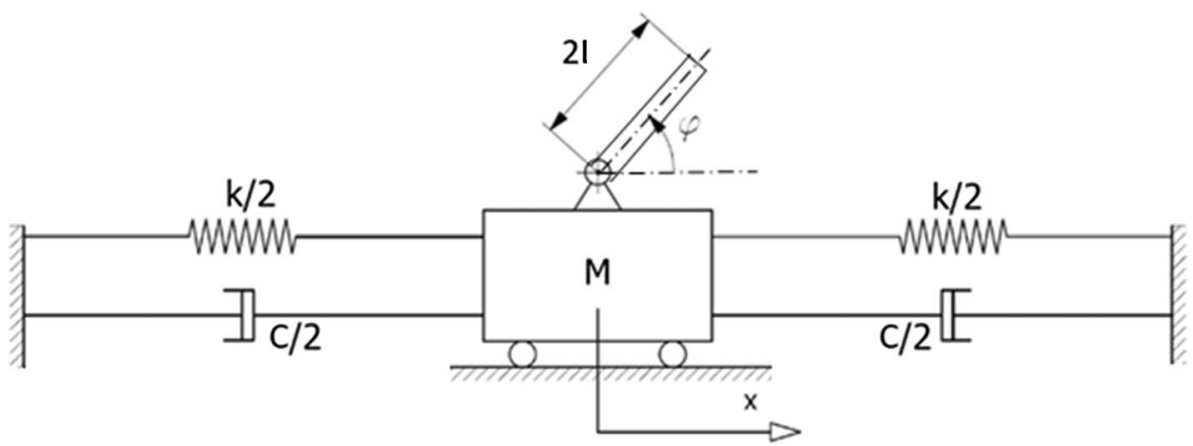


Fig. 2 The simulation results: a cart displacement amplitude at resonance; $\mathbf{b}$ mechanical energy of the rotating rod mass $m$ versus energy of the mass $M$
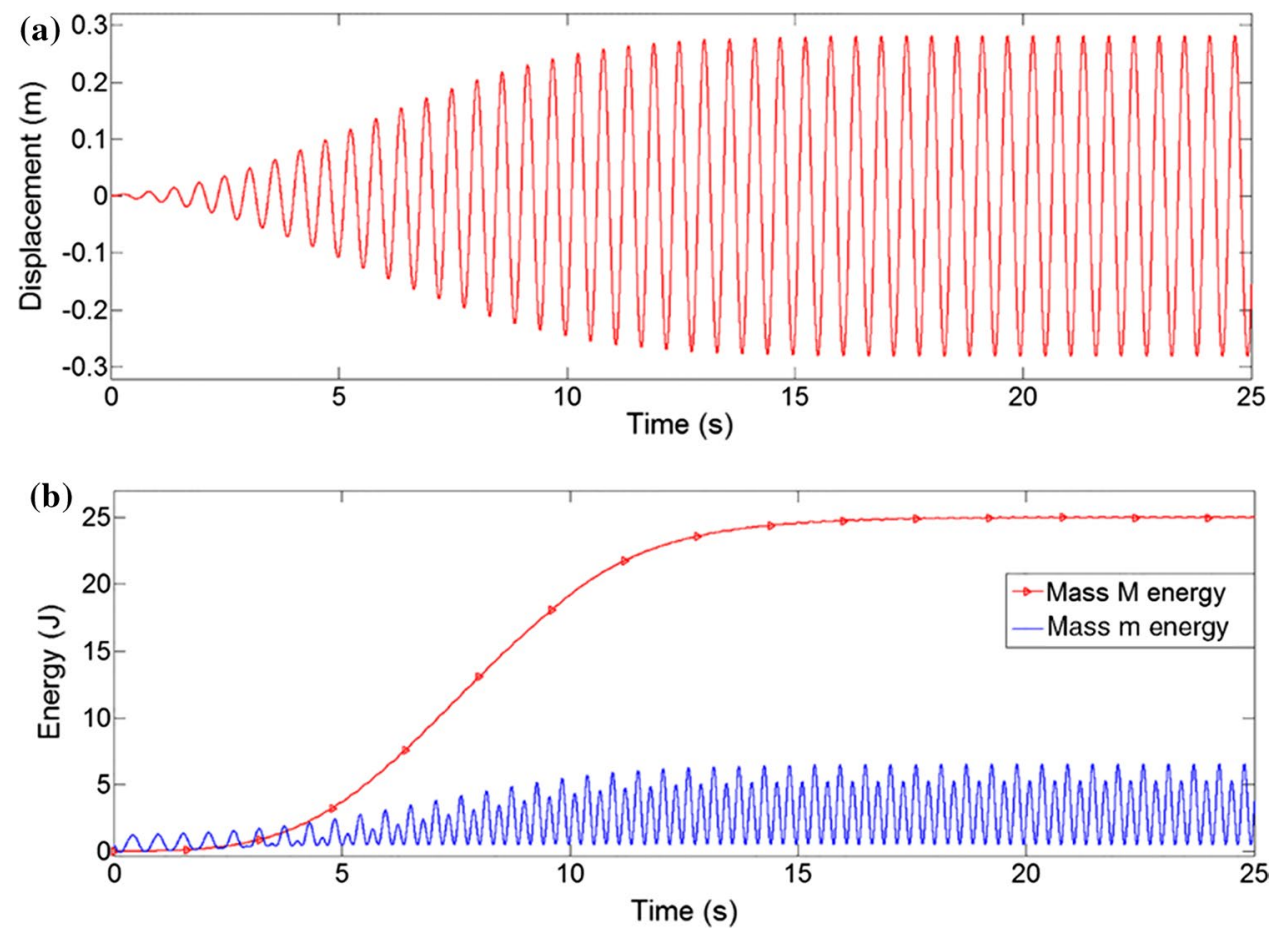

Figure $2 b$ presents the energy of the rotating rod mass in comparison with the energy of the cart mass at resonance. It can be seen that in the state of resonance, the energy of the oscillating cart mass is several times higher than this of the rotating rod mass.

Energy absorbed in the oscillating mass during one oscillation at steady-state conditions of resonance is equal to the dissipation energy:

$E_{\mathrm{dis}}=c A_{\max }^{2} \omega_{\mathrm{d}}^{2} \int_{0}^{T} \cos ^{2}\left(\omega_{\mathrm{d}} t-\theta\right) \mathrm{d} t=\pi c \omega_{\mathrm{d}} A_{\max }^{2}$

where $A_{\max }$-maximum amplitude, $\omega_{d}=\sqrt{\omega_{0}^{2}-2 \xi^{2}}-$ damped natural frequency, $\omega_{0}$-undamped natural frequency, $\mathrm{c}$-damping coefficient and $\xi$-damping ratio.

The maximal energy accumulated in resonance is defined by the sum of the kinetic and potential energy of the oscillating mass:

$E_{\text {acmax }}=\frac{1}{2} k A_{\max }^{2}=\frac{1}{2} m \omega_{\mathrm{d}}^{2} A_{\max }^{2}$

The maximal amplitude at resonance can be established from:

$A_{\text {max }}=\frac{F_{0}}{c \omega_{d}}$

where $F_{0}$-amplitude of the excitation force
Maximal energy which can be accumulated at resonance can be established after (8) will be implemented in (9) from:

$E_{\text {acmax }}=\frac{k F_{0}^{2}}{2 c^{2} \omega_{\mathrm{d}}^{2}}=\frac{m F_{0}^{2}}{2 c^{2}}$.

The maximal energy accumulated in the oscillator depends on the stiffness, mass, damping of the system and the excitation force. With increasing excitation force $F_{0}$, amplitude of the energy accumulated in the resonance increases. On the other hand, with the increasing of damping and resonance frequency the energy accumulated at the resonance decreases relatively strong.

It should be noted that the presented analytical model does not take into account possible nonlinearities occurring in the system, e.g., nonlinear friction between the guide and the mass. These nonlinearities, however, are typically too small to change the behavior of the considered system during resonance.

\section{Sequential extraction of the energy at resonance}

The energy of the oscillator in resonance can be extracted sequentially. In Fig. 3, waveforms representing the cart displacement, energy of the cart and energy of the rotating rod mass in the course of sequential extraction of the cart energy are shown. When the external load is applied to the oscillator, the displacement amplitude decreases to a minimum 
Fig. 3 Sequential extraction of energy at resonance: a displacement of the cart; $\mathbf{b}$ energy of the cart compared to energy of the rotating rod mass (simulation results)

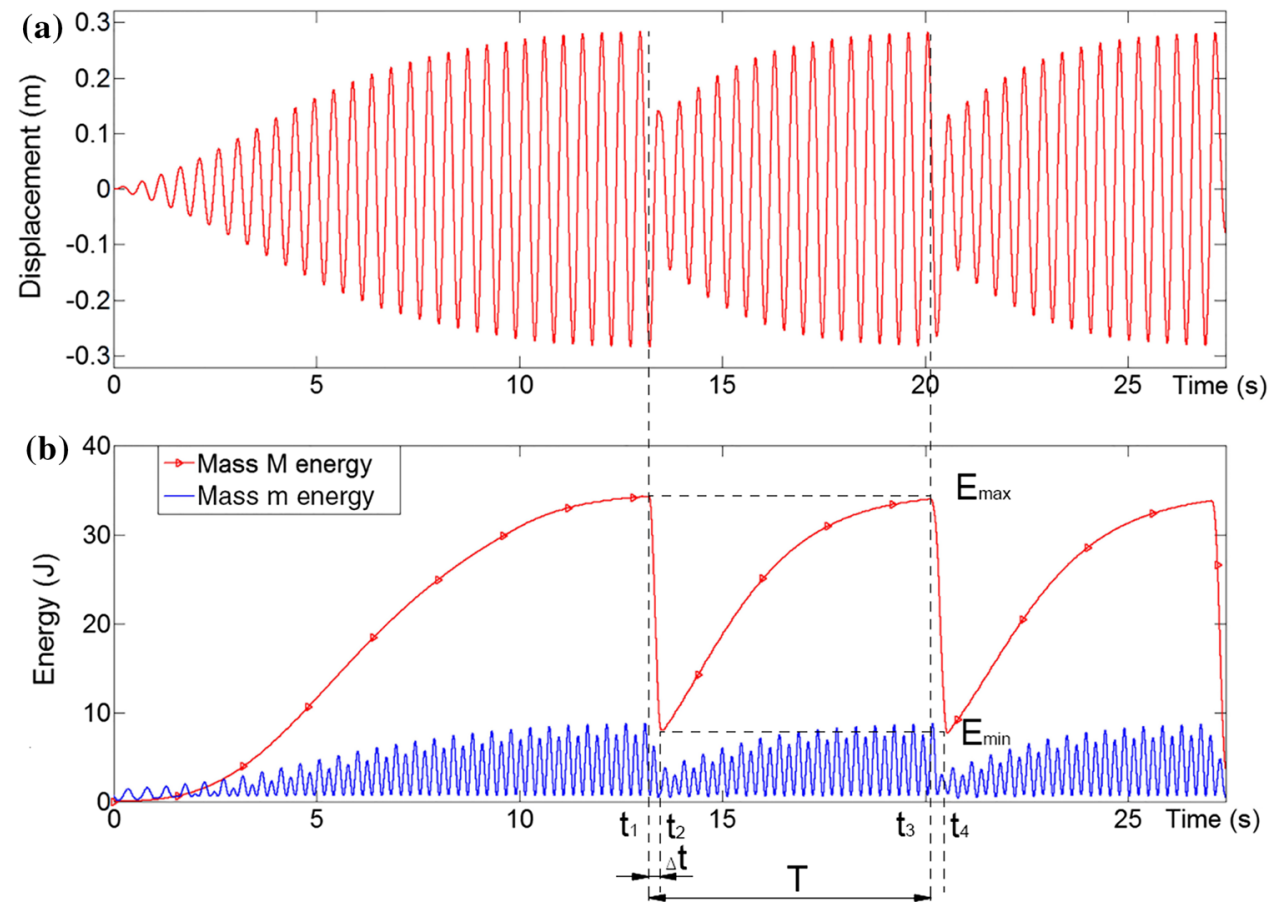

value and subsequently after a defined time and depending on the damping, increases to its maximum value.

At time $t=0$ (Fig. 3), the oscillator starts to vibrate with the resonance frequency. At time $t_{1}$, the displacement of the cart achieves its maximum amplitude and the energy takeup begins until $t_{2}$, at which time the reception of energy is stopped. From $t_{2}$ to $t_{3}$, restoration of displacement amplitude of the cart occurs up to the maximum value and the process is further repeated with a period $T$ equal to the restoration time which is dependent on the damping constant and the angular frequency of the oscillator. The energy $E_{\mathrm{R}}=E_{\max }$ $-E_{\min }$ can be extracted within the time interval $\Delta t=t_{2}-t_{1}$. The maximum energy extracted is the higher the shorter is the interval $\Delta t$. The process of steady energy transfer to the rotating rod mass will be affected due to the extraction of the energy. After a decrease in the amplitude of energy changes in interval $\Delta t$, the energy supplied increases up to a maximum value and the energy of the cart reaches its maximal value.

\section{Experimental investigations}

Figure 4 shows a single-mass oscillator created for the purpose of experimental verification of theoretical considerations. The device comprises a mass $M(1)$, linear bearing system (2), set of two springs (3) and frame (4) attached to a solid base. Excitation force is introduced by rotation of an excitation mass (5) coupled with a DC motor (6) set on top of the mass $M$. The drive angular velocity is controlled by voltage applied to DC motor. The oscillation amplitude of the mass $M$ increases significantly when the angular frequency of the rotating rod mass gets closer to the resonance frequency of the system. The position of the cart was assessed with an optical displacement sensor.

In order to extract the power accumulated in the cart mass $M$, a test rig with a brake system shown in Fig. 4 was used.

A rack-pinion system (7) was connected to the cart. The pinion was set on a shaft with bearings supported by a base. The other end of the shaft was equipped with a clamping brake system (8). The clamp (9) was controlled manually by the operator. A full-bridge strain gauge (10) was placed on the pinion shaft between the rack and brake disk. The pinion shaft was suspended on a bearing assembly (11).

The sequential extraction of energy has been investigated experimentally. The torque measurements on the setup shown in Fig. 4 with a full-bridge strain gauge (10) were taken. Displacement of the cart corresponded to changes of angular velocity of a pinion which was calculated based on the linear velocity of the pinion rack and the pitch diameter of the pinion. Both values were used for calculation of mechanical power according to Eq. (13).

To determine the power $P_{\mathrm{EM}}(t)$ supplied to the oscillator, voltage $u_{\mathrm{A}}(t)$ and current $i(t)$ on the electrical motor were measured to obtain

$P_{E M}(t)=u_{A}(t) \cdot i(t)$. 
Fig. 4 Experimental test stand a test rig, b load application system

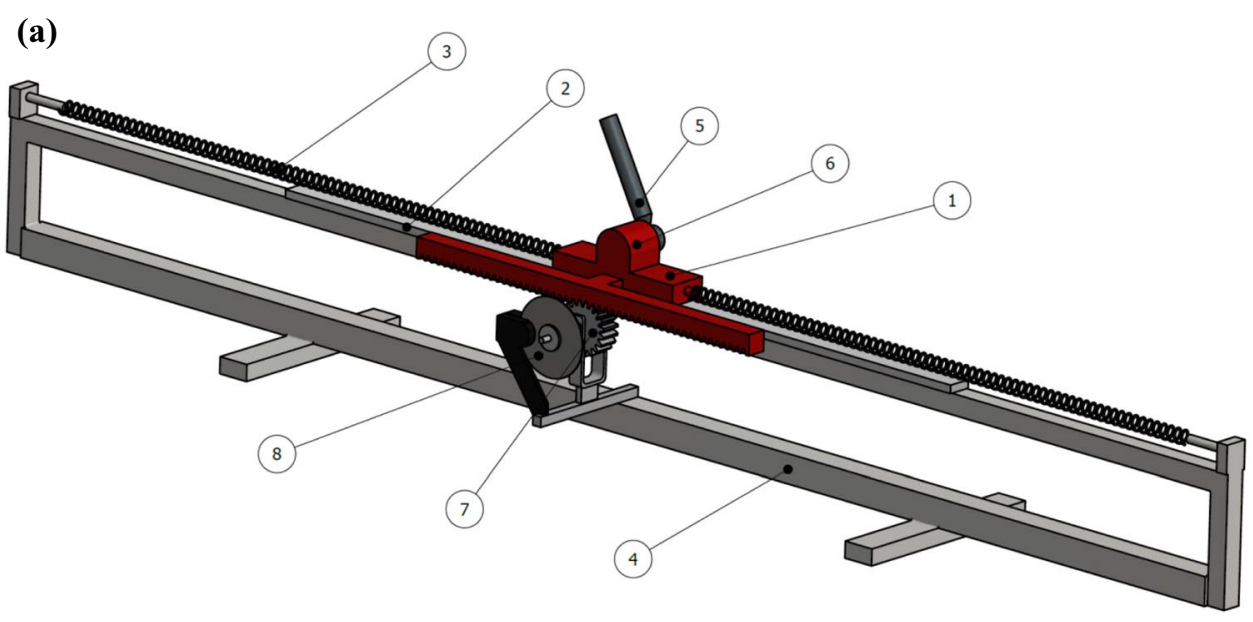

(b)

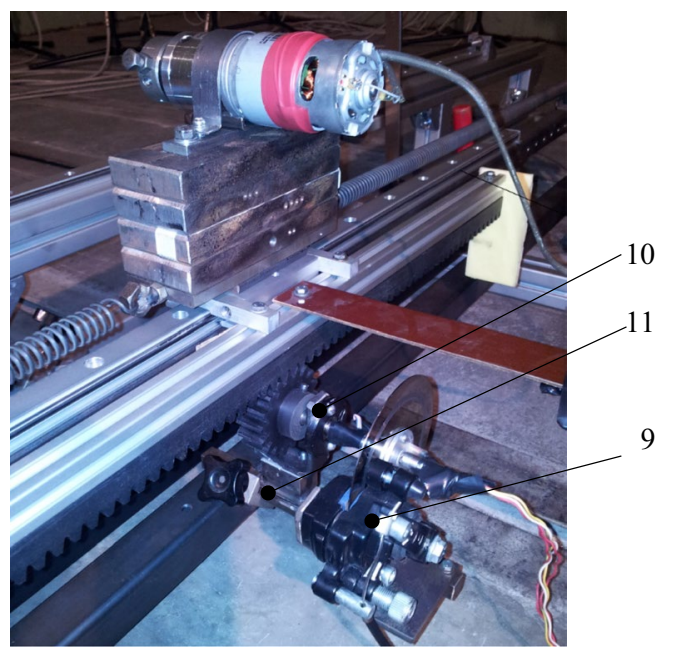

A $1 \mathrm{ohm}$ resistor was connected to the electrical motor supply line to calculate the current from:

$i(t)=\frac{u_{B}(t)}{R}$

where $u_{\mathrm{B}}(\mathrm{t})$ is the voltage on the resistor $R=1 \mathrm{ohm}$.

The efficiency of the motor was determined by measurements of angular velocity and external load, in similar conditions to those at resonance state of the experimental oscillator presented in Fig. 4. The energy $E_{\mathrm{IN}}$ supplied to the oscillator was calculated as

$E_{\mathrm{IN}}=\eta_{\mathrm{EM}} \int P_{\mathrm{EM}} \cdot \mathrm{d} t$

where $\eta_{\mathrm{EM}}$ is the efficiency of the electric motor.

During the measurement, the oscillator was excited with the resonance frequency. When the amplitude of oscillation reached its highest value, an external load was applied by the brake clamp. The load torque was measured by a fullbridge strain gauge installed between the pinion and the brake (Fig. 4). The angular velocity of the shaft was established from the linear velocity of the cart and the radius of the pinion. The instantaneous mechanical power extracted from the oscillator was calculated from

$P_{M}(t)=T(t) \cdot \omega(t)$.

Cart velocity was obtained as the derivative of the displacement acquired from measurements. Information about spring stiffness, mass values, cart displacement and velocity was used to compare the energy of the cart and the rotating rod mass. All calculations were performed in the MATLAB and Simulink environments.

Results from measurements regarding the amplitude were compared with the simulation results, and good coincidence has been found [19].

The load can be applied in the form of repeatable pulses applied for a short time. Figure $5 \mathrm{~b}$ presents the comparison of power received by the brake system and the power demand of the gear motor. In the case of the pulse-like sequence, the change in amplitude of the displacement of 
Fig. 5 Sequential extraction of the energy at resonance-measurement; a mass $m$ displacement, b mechanical power supplied to the oscillator versus mechanical power received from the oscillator
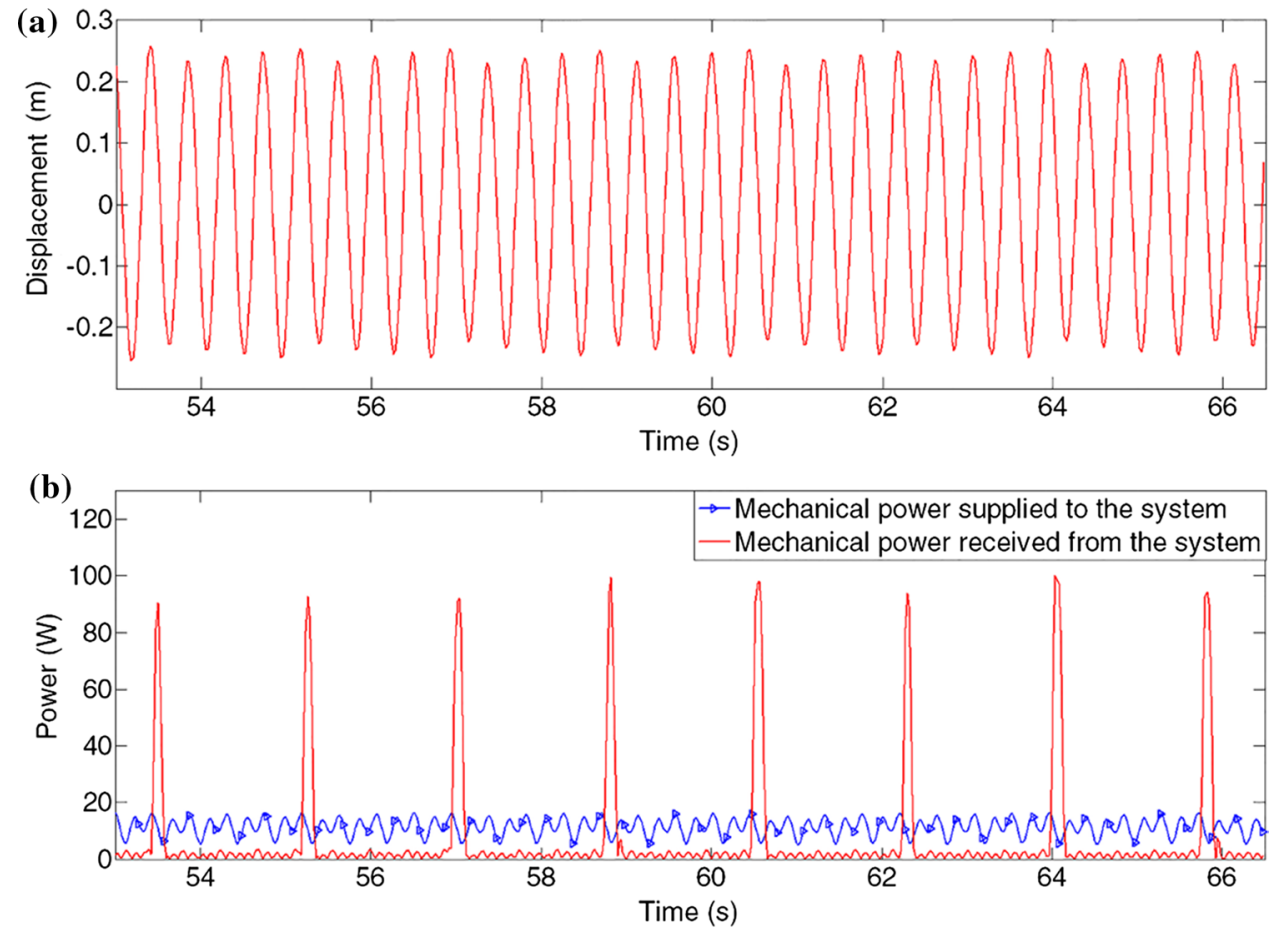

the cart is relatively small (Fig. 5a). After release of the load, the amplitude starts to rebuild up to the maximum resonance value after every $2 \mathrm{~s}$.

A decrease in displacement amplitude of the cart mass is strongly related to the duration time of the load pulse. For longer pulse duration times, oscillations of the cart drop strongly. The displacement of the cart should therefore not decrease below certain value in one cycle to achieve a short restoration time of maximal amplitude at resonance.

The torque measurements according to the setup in Fig. 4, with a full-bridge strain gauge (10), have been taken. Displacement of the cart shown in Fig. 6a corresponds to the changes of pinion angular velocity. The angular velocity of the pinion was calculated based on the linear velocity of the pinion rack and the pitch diameter of the pinion. Both values were used for the calculation of mechanical power $\mathrm{P}_{M}$ according to Eq. (13).

The load was applied in the form of repeatable impulses lasting a short period of time. Figure $5 \mathrm{~b}$ presents the comparison of power received by the brake system and the power at the gear motor. In the case of the impulse-like sequence, the change in amplitude of the displacement of the cart is relatively small (Fig. 5a). After release of the load, the amplitude starts to rebuild to the maximum resonance value after every $2 \mathrm{~s}$.

A decrease in displacement amplitude of the cart mass is strongly connected with the duration time of the load impulse. For a longer duration time of the load impulse, the oscillations of the cart drop strongly. The displacement of the cart should therefore not decrease below the certain value in one cycle to achieve a short restoration time of maximal amplitude at resonance.

The load on the pinion can be also considered electrically by means of an electric generator. In this case, the output power will be measured as the electric power of the generator.

\section{Prototype of the resonance punching press}

The major element of the resonance press (Fig. 6) is the resonant block, set on a linear bearing system attached to the housing of the device. The resonant block is also connected to the housing by a set of springs. Oscillations of the main element can be induced by means of inertial forces caused by the rotating mass or due to hydraulic, pneumatic or electric actuator action. Energy accumulated in the resonant block can be applied to the punching tool by activating the clutch control system, which is triggered at set oscillation amplitude. After performing the punching operation, the clutch is automatically disengaged in order to increase the vibration amplitude to needed values and repeat the punching process periodically. The energy supplied to the resonant block will be used to cover the losses caused by frictional forces. The time needed to rebuilt the vibration amplitude and to accumulate the maximal energy is dependent mainly from the excitation force amplitude and system damping. 
Fig. 6 a Prototype of the resonance punching press: a $3 \mathrm{D}$ model: 1 -punching device, 2-rotational mass, 3-clutch engage switch, 4-punch clutch triggers, 5-set of springs, 6-electric motor, 7-gearbox, 8-housing, 9-resonant block and 10 - bevel gears; $\mathbf{b}$ a photograph of the machine [20]

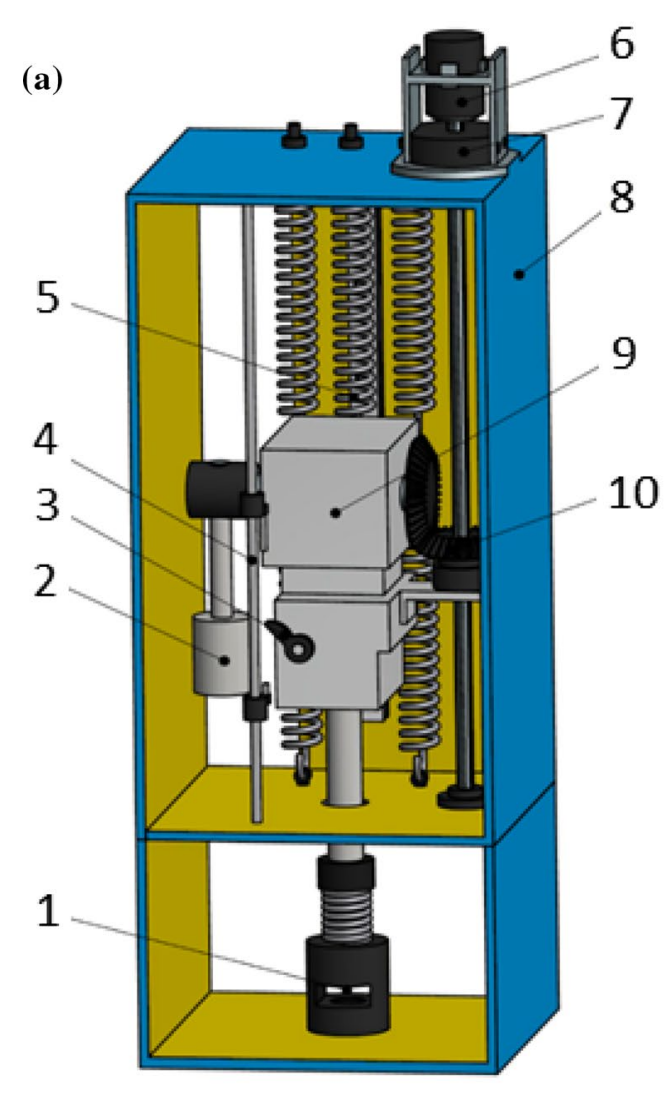

(b)

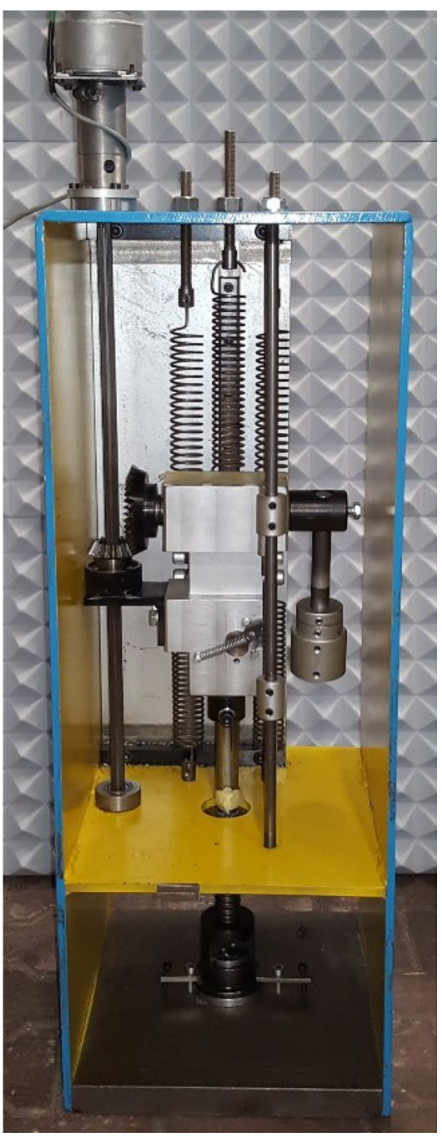

Strong decrease in the resonant block vibration amplitude could significantly increase the time needed to rebuild the sufficient oscillations similar as in the case of flywheel torque in eccentric machines the decrease should not reach more than $10 \%$.

In most impact operating machines, periodic reception of energy occurs during the work processes, e.g., pressing operations or punching holes in sheet metal. In eccentric presses, the energy needed to perform the punching process is stored in a flywheel. The mechanical resonance can be also used in other machines such as stamping machines, impact forging hammers and drills.

Figure $6 \mathrm{a}$ shows a 3D model and of a prototype resonance press. The resonant block (9) oscillations are induced by means of a rotational mass (2). Input power is supplied by an electric motor (6) via a reducing gearbox (7). The punching procedure is activated at resonance of the block when the kinetic energy reaches maximum values. The punch is coupled with the resonant block via a controlled clutch (3) which engages only during the punching process. Clutch (3) activation and deactivations take place at set oscillation amplitude, controlled by means of mechanical triggers (4).

The dynamical model of the resonance press in Adams software is shown in Fig. 7. Based on this model, the main parameters of the prototype of the resonance press have been selected.

The force required to punch a hole with a diameter $d_{1}$ in a sheet steel with thickness $t_{1}$ can be calculated from [20]:

$F_{\mathrm{s}}=A \tau_{\mathrm{u}}=\pi d_{1} t_{1} \tau_{\mathrm{u}}=9.420 \mathrm{kN}$

where $A$ is the area sheared; $d_{1}=10 \mathrm{~mm}$-diameter of the punched hole; $t_{1}=1 \mathrm{~mm}$-thickness of sheet metal; and $\tau_{\mathrm{u}}=300 \mathrm{MPa}$ - the ultimate shear stress.

For a resonant block with mass of $12 \mathrm{~kg}$ suspended on 4 springs with total stiffness of $3980 \mathrm{~N} / \mathrm{m}$, the natural frequency of the oscillator would be $2.8 \mathrm{~Hz}$. The maximum amplitude of oscillations $A_{\max }$, which depends on damping, will be assumed to be $0.11 \mathrm{~m}$.

The force that can be achieved with the resonance punching press described above is:

$F_{\mathrm{s}}^{\prime}=\frac{E}{t_{1}}=22.2 \mathrm{kN}$.

The value is about 2 times higher than this required to punch the hole $(9420 \mathrm{~N})$. It is important that the amplitude of oscillations of the resonant block after a duty cycle does not decrease below a certain value for which the regaining of maximum oscillation amplitude takes a longer period 


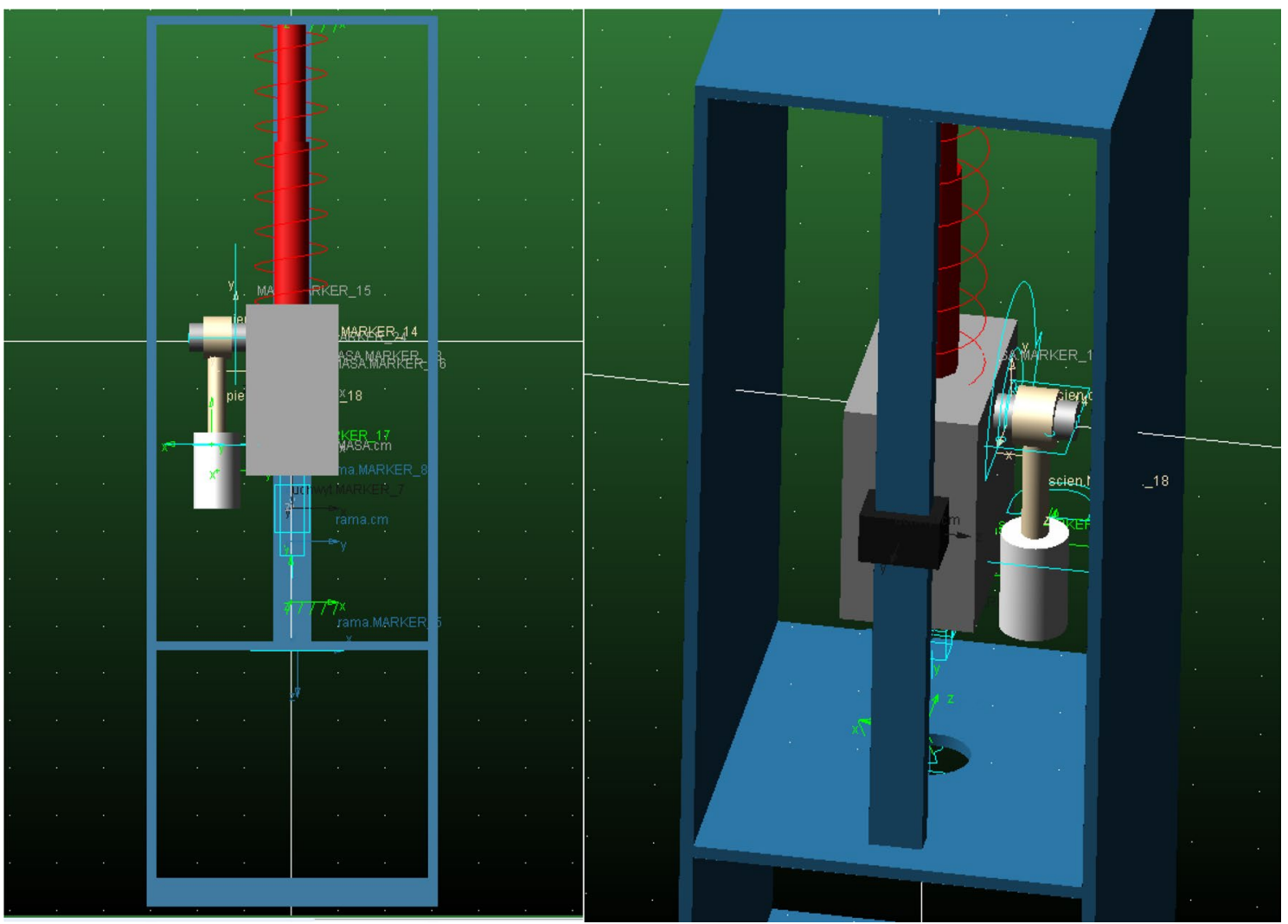

Fig. 7 Model of the resonance press in Adams

of time. A similar rule is valid in the case of flywheel eccentric presses where the velocity fluctuation ratio of the flywheel needs to be below $10 \%$ [22].

In conventional presses, the energy needed to perform the punching is stored in the flywheel. Assuming a ringshaped mass flywheel, the kinetic energy is defined as:

$E_{\mathrm{kfw}}=\frac{1}{2} I \omega^{2}=\frac{1}{2} m \omega^{2} r^{2}$.

Equations (18) and (9) show that the energy of the oscillator and flywheel is equal when $r=A_{\max }$; both flywheel and oscillator have equal masses, and the angular velocities of excitation elements are the same for both cases. Average energy supplied to the oscillator at steady state resonance conditions is equal to the dissipated energy and is several times lower than the energy accumulated in the oscillator, as shown in Fig. 8. Energy applied to the flywheel is defined as the kinetic energy of the flywheel.

Figure 8 presents the comparison of flywheel and oscillator energy increase in time. It can be seen that the oscillator energy rises faster and presents higher values than the flywheel until the time $t_{0}$.
Fig. 8 Energy comparison: oscillator versus flywheel, $t_{0}$ time point of equal energy [21]

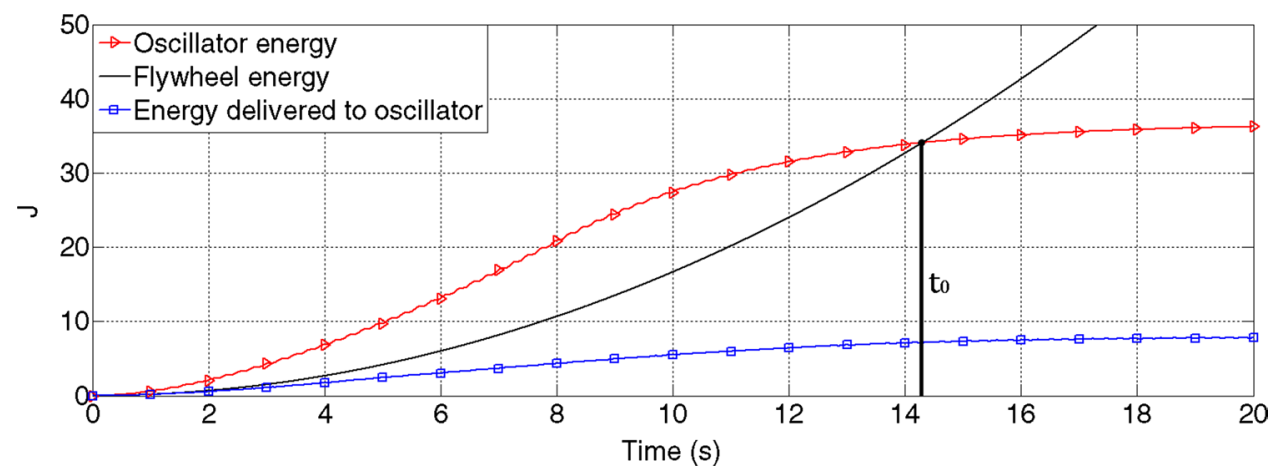


Analysis of Fig. 8 can also lead to the observation that the average energy supplied to the oscillator is many times smaller than the energy stored in the oscillator.

At resonance, the inertia forces of the oscillator are balanced by the forces generated on springs, due to that the electric motor is used mainly to cover friction forces in the bearing assembly. In the case of the flywheel, the punching procedure is required to overcome the process forces, inertia forces of the flywheel and the friction in the bearings of the flywheel.

The power supplied to the prototype resonance press (Fig. 6) was confronted with the power supplied to a conventional eccentric press (Fig. 9) designed for similar punching purposes and operating parameters.

The flywheel in the eccentric press (Fig. 9) drive consists of an electric motor and belt transmission. Mechanical power generated on the electric motor is transmitted to the flywheel set on an eccentric shaft that drives a crank connected to the ram assembly set on a slider.

During experimental comparison of both presses, process parameters such as punch diameter, material type, sheet thickness as well as course of punching were the same. Processed sheet was steadily displaced in the punching device allowing sequential operation and providing full contact area of die and sheet at each punch.

Comparison between two types of presses was possible by section of work parameters for both presses so that the energy the accumulated in the resonance press is comparable to the energy of flywheel in conventional eccentric press.

Energy accumulated in the resonant block at maximum amplitude $A \max =0.11 \mathrm{~m}$ is as follows:

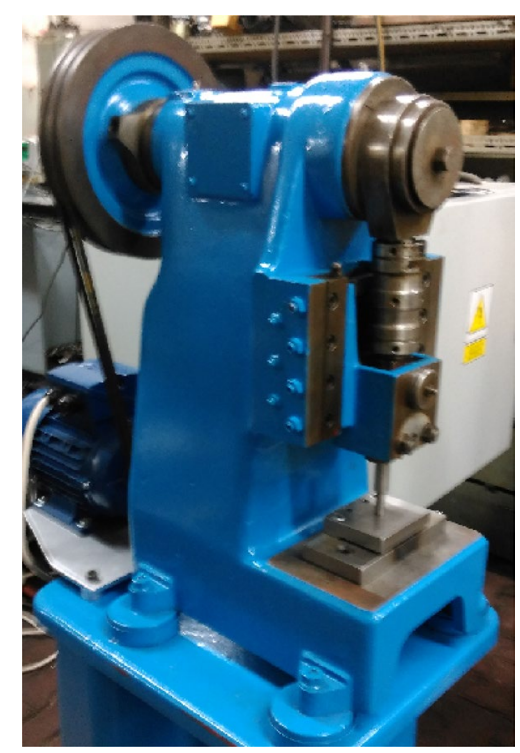

Fig. 9 Conventional eccentric press [20]
Table 2 Parameters of eccentric and resonance press

\begin{tabular}{lll}
\hline Press type & Eccentric press & Resonance press \\
\hline Operating frequency $(\mathrm{Hz})$ & 2.8 & 2.9 \\
$\begin{array}{l}\text { Motor speed (rpm) } \\
\text { Mass of flywheel/resonant block }\end{array}$ & 177 & 174 \\
$\quad(\mathrm{~kg})$ & & 12 \\
$\begin{array}{l}\text { Radius of the flywheel (m) } \\
\text { Amplitude of the oscillations (m) }\end{array}$ & 0.126 & - \\
Power demand (W) & 169 & 0.11 \\
\hline
\end{tabular}

$E_{\mathrm{r}}=\frac{1}{2} m_{\mathrm{r}}(2 \pi f)^{2} A_{\max }^{2}=0.5 \cdot 12 \cdot(2 \pi \cdot 2.9)^{2} \cdot 0.11^{2}=24 \mathrm{~J}$.

The kinetic energy stored in the flywheel with a radius of $\mathrm{r}=0.126 \mathrm{~mm}$ is;

$$
\begin{aligned}
I & =\frac{1}{2} m_{k} \cdot r^{2}=0.5 \cdot 18 \cdot 0.126^{2}=0,143 \mathrm{~kg} \mathrm{~m}^{2} \\
E_{k} & =\frac{1}{2} I \omega^{2}=0.5 \cdot 0.143 \cdot(2 \pi \cdot 2.8)^{2}=22.2 \mathrm{~J} .
\end{aligned}
$$

The DC motor power used in the resonance press was defined as the root mean square current and voltage values measured motor terminals:

$P=U_{r m s} \cdot I_{r m s}$

where $U_{\text {rms }}$-effective voltage value; $I_{\text {rms }}$-effective current value.

The power supplied to the $\mathrm{AC}$ motor driving the eccentric press was measured with a three-phase wattmeter. Table 2 presents the power demand in the case of each press. It can be seen that the power consumption in the case of the resonance press is 2.86 times lower than in the case of the eccentric press. This surprisingly high result should be treated with caution; more investigations and further tests are needed due to the fact that the motor used in the eccentric press has been not optimized regarding the lowest energy consumption.

Energy losses in the eccentric press are greater due to the rotary to reciprocating motion conversion at the flywheel to punch drive chain. The comparison of power losses in both presses is shown in Fig. 10.

The comparison shows that the case of an eccentric press has more potential places where energy losses occur. The design complexity of the eccentric press is definitely much higher than this characterizing the resonance press.

\section{Conclusions}

An analysis of energy waveforms observed in both simulations and experiments leads to the following conclusions. At a steady state of resonance, the total energy absorbed by the 
Fig. 10 Comparison of energy losses: a eccentric press and $\mathbf{b}$ resonance press

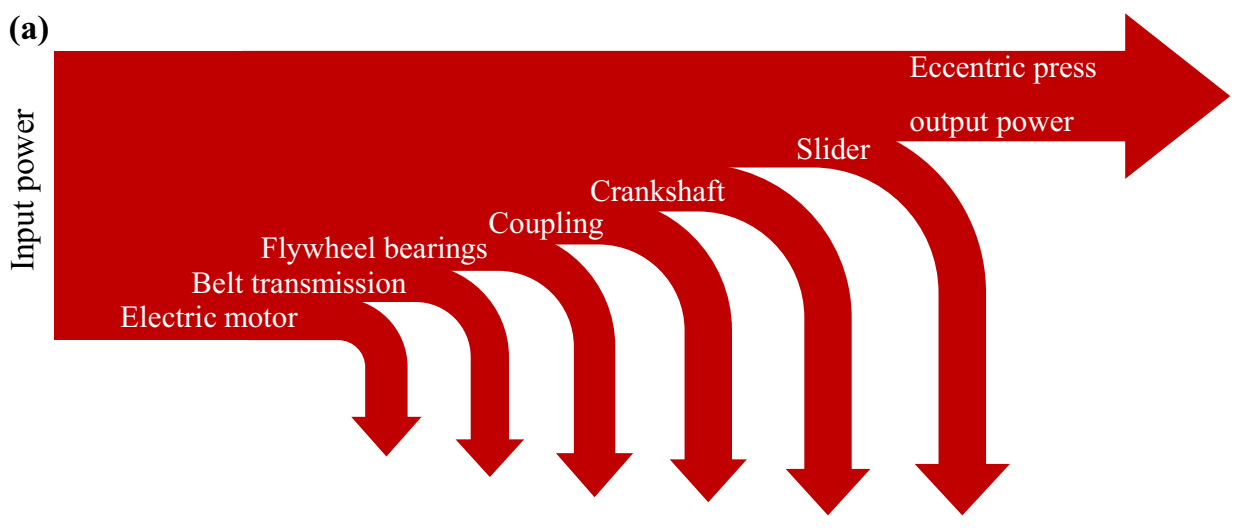

(b)

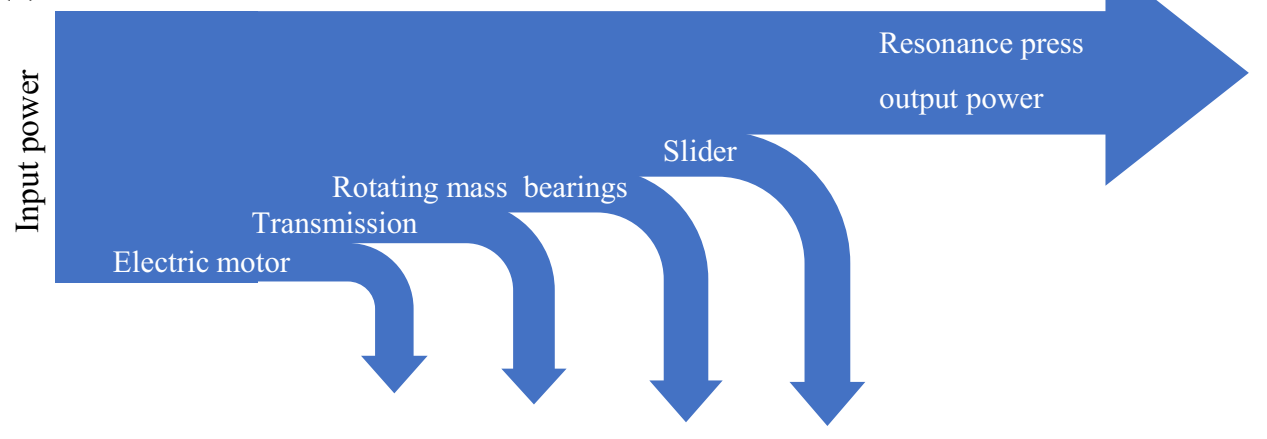

oscillating mass is equal to the energy lost due to damping. The energy is accumulated in the oscillator, as in the case of the flywheel, and can be several times higher than the energy supplied continuously to the system. Sequential extraction of energy, in form of high-value force impulses, is possible in the case of resonance systems. Force pulse values and the power gain from the oscillator at resonance are defined by operating frequency and damping ratio.

The advantage of using the phenomenon of mechanical resonance in resonance press has been presented.

The phenomenon of oscillator energy accumulation has been compared with conventional energy accumulation process in a flywheel. Investigations show that the accumulation at resonance conditions is higher and proceeds faster than in the case of a flywheel with similar system parameters such as energy storing device mass, rotational speed or vibration frequency and radius or amplitude of vibration.

The durability of the spring elements that are used in the resonance press should be higher than in conventional spring elements. This can be achieved by using improved materials for springs and their proper fastenings. Steel springs can also be replaced in some applications by pneumatic springs.

The energy required to excite an oscillator to mechanical resonance is many times lower than the energy that must be delivered to a flywheel of the same mass and angular speed. The mass of the equivalent oscillator is less than the mass of flywheel, especially for lower operating frequencies. This feature can be used for minimization of mass of some drive systems. In resonance systems, the load from the inertia forces is equalized by the spring force and the supplied power is used to compensate the workload and the friction resistance. For this reason, dynamic forces in individual components of the system will be smaller, particularly the forces on bearings which may affect the overall service life of any mechanical system. The significantly lower power demand measured on prototypes of the resonance press compared to an equivalent eccentric press has been confirmed. The presented method of extracting energy accumulated during the resonance in impact machines is innovative and has not been used in practice so far.

The method presented in this paper differs from that used in energy harvesting. During harvesting of energy from vibrations, the oscillator is forced through springs and in this method there is a direct harmonic excitation applied to the oscillator mass with the resonance frequency.

Further research will be focused on optimizing of parameters of impact machines as such, parameters of systems designed to control them, and their advantages over conventional machines as far as the energy consumption is concerned. 


\section{Compliance with ethical standards}

Conflict of interest The authors declare that they have no conflict of interest.

Ethical approval This manuscript has not been published by another journal.

Open Access This article is licensed under a Creative Commons Attribution 4.0 International License, which permits use, sharing, adaptation, distribution and reproduction in any medium or format, as long as you give appropriate credit to the original author(s) and the source, provide a link to the Creative Commons licence, and indicate if changes were made. The images or other third party material in this article are included in the article's Creative Commons licence, unless indicated otherwise in a credit line to the material. If material is not included in the article's Creative Commons licence and your intended use is not permitted by statutory regulation or exceeds the permitted use, you will need to obtain permission directly from the copyright holder. To view a copy of this licence, visit http://creativecommons.org/licenses/by/4.0/.

\section{References}

1. Crocker MJ. Handbook of noise and vibration control. Hoboken: Wiley; 2007. p. 528-45.

2. Harris CM, Piersol AG. Shock and vibration handbook. New York: McGraw-Hill; 2002.

3. Crawford A. Simplified handbook of vibration analysis, vol. 1. Knoxville, USA: Computational Systems; 1992.

4. Aguiar R, Weber HI. Development of vibroimpact device for the resonance hammer drilling. In: Proceedings of the XII international symposium of dynamic problems of mechanics, Feb 26Mar. 02; 2007.

5. Despotovic Z, Ribic A. A comparison of energy efficiency of SCR phase control and switch mode regulated vibratory conveying drives. In: IX symposium industrial electronics, Banja Luka, Nov 01-03; 2012.

6. Plooij MC, Wisse M. A novel spring mechanism to reduce energy consumption of robotic arms, intelligent robots and systems (IROS). In: IEEE/RSJ international conference, 2901-2908, 2012.

7. Baek S, Ma K, Fearing R. Efficient Drive of flapping- wing robots. In: IEEE/RSJ international conference on intelligent robots and systems, St. Louis, USA, Oct 11-15; 2009.
8. Glynne-Jones P, Tudor MJ, Beeby SP, White NM. An electromagnetic, vibration-powered generator for intelligent sensor systems. Sens Actuators A. 2004;110:344-9.

9. Stephen NG. On energy harvesting from ambient vibration. J Sound Vib. 2006;239:409-25.

10. Lei Z, Xiudong T. Large-scale vibration energy harvesting. J Intell Mater Syst Struct. 2013;24(11):1405-30.

11. Colins L. Harvesting for the world: energy harvesting techniques. IEEE Power Engineer. 2006;20:34-7.

12. Zuo L, Scully B, Shestani J, Zhou Y. Design and characterization of an electromagnetic energy harvester for vehicle suspensions. Smart Mater Struct. 2010;19:1-10.

13. Goldner RB, Zerigian P. A preliminary study of energy recovery in vehicles by using regenerative magnetic shock absorbers, SAE technical paper series 2001-01-2071;2001.

14. Kammer AS, Olgac N. Delayed-feedback vibration absorbers to enhance energy harvesting. J Sound Vib. 2016;363:54-67.

15. Han X, Xu W, Sabu J. A multi-degree of freedom piezoelectric vibration energy harvester with piezoelectric elements inserted between two nearby oscillators. Mech Syst Signal Process Vol. 2016;68-69:138-54.

16. Horodinca M, Saghedin NE. Experimental investigations of power absorbed at mechanical resonance. Exp Tech SEM. 2011; 1-11.

17. Aldraihem O, Baz A. Energy harvester with a dynamic magnifier. J Intell Mater Syst Struct. 2011;22(6):521-30.

18. Heo YJ, Lee WC, Kim T, Cho Y. Active micromechanical motion amplifiers using the mechanical resonance modulated by variable stiffness springs. Sens Actuators A. 2012;180:97-104.

19. Fiebig W, Wrobel J. Simulation of energy flow at mechanical resonance. In: 22nd ICSV conference, Florence, Italy, July 12-16; 2015.

20. Fiebig W, Wrobel J. Use of mechanical resonance in impact machines. In: Maia N, Dimitrovová Z (eds) The 14th international conference on vibration engineering and technology of machinery VETOMAC XIV, Lisbon, Portugal, September 10-13, 2018; 2018, p. 1-6.

21. Fiebig W, Wrobel J. Use of mechanical resonance in machines drive systems. In: 24th ICSV conference, London, UK, July 23-27; 2017.

22. Khurmi RS, Gupta JK. Theory of machines 14th edn, chapter 16: turning moment diagrams and flywheel, S. Chand \& Co. Ltd., New Delhi; 2005, p. 565-611.

Publisher's Note Springer Nature remains neutral with regard to jurisdictional claims in published maps and institutional affiliations. 